\title{
The Bergman kernel for the Vekua equation
}

\author{
Hugo M. Campos ${ }^{\mathrm{a}}$, Vladislav V. Kravchenko ${ }^{\mathrm{b}}$

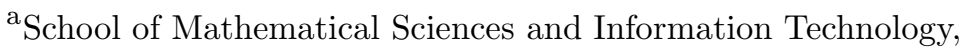 \\ Yachay Tech, Urcuqui 100119, Ecuador \\ email: hfernandes@yachaytech.edu.ec \\ ${ }^{\mathrm{b}}$ Regional mathematical center of Southern Federal University, \\ Bol'shaya vadovaya, 105/42, Rostov-on-Don, 344006, Russian Federation, \\ on leave from the Department of Mathematics, CINVESTAV del IPN, \\ Unidad Queretaro, Libramiento Norponiente No. 2000, \\ Fracc. Real de Juriquilla, Queretaro, Qro. C.P. 76230 MEXICO \\ e-mail:vkravchenko@math.cinvestav.edu.mx*
}

September 6, 2021

\begin{abstract}
The paper extends some well-known results for analytic functions onto solutions of the Vekua equation $\partial_{\bar{z}} W=a W+b \bar{W}$ regarding the existence and construction of the Bergman kernel and of the corresponding Bergman projection operator.
\end{abstract}

\section{Introduction}

The Bergman kernel is a useful tool in complex analysis with important applications in differential geometry, in theory of analytic and harmonic functions and in the study of conformal mappings [3] (see also [10] for new results and extensions to several complex variables). The idea behind its construction is rather simple. The space of square integrable analytic functions on a planar domain (called Bergman space) is associated with a kernel that allows the reproduction of any function in the Bergman space.

${ }^{*}$ H. M. Campos acknowledges the support by Yachay Tech, Ecuador and CONACYT, Mexico via the research project CB 2013-222478F. V. V. Kravchenko acknowledges the support by CONACYT, Mexico via the research project CB 2016-284470. 
This kind of reproducing property was the base of important generalizations. On the one hand, S. Bergman and his collaborators developed a method for solving boundary value problems for elliptic pde's with variable coefficients, based on the construction of some appropriate reproducing kernels [4]. On the other hand, analogous reproducing properties appeared in the work of other mathematicians of that time (and even earlier) in the study of other functional spaces. In [1] the abstract theory of reproducing kernel Hilbert spaces (RKHS) was developed. The Bergman-type kernels are concrete examples of RKHS. For the main results, applications and historical facts about the RKHS theory see [1], 2, [14] and [15]. Despite the fact that the RKHS theory is well developed, when a concrete reproducing kernel is found, special properties of the particular kernel and new connections with different branches of analysis arise.

The goal of this paper is to establish the existence and some first properties of the Bergman kernel for the Vekua equation. The paper is organized as follows. In Section 2 we present some well known properties of solutions of the Vekua equation (1) needed throughout the paper and in Section 3 we define and study the corresponding Bergman space $H^{2}(\Omega)$. Contrary to the case of the Bergman space of analytic functions, $H^{2}(\Omega)$ is a real linear space (in general, not complex). By introducing a suitable scalar product we realize that $H^{2}(\Omega)$ is a Hilbert space and establish useful convergence properties. Using this, we show in Section 4 that the real and the imaginary parts of the pointwise evaluation are continuous linear functionals on $H^{2}(\Omega)$. This leads first to the existence of the Bergman kernel in $H^{2}(\Omega)$ and second to a realization of a reproducing property in the space $H^{2}(\Omega)$. Finally, in Section 5 we provide a method for constructing the Bergman kernel in terms of any countable orthonormal basis in $H^{2}(\Omega)$. Moreover, we prove that such countable orthonormal basis always exists and provide some suggestions for its construction. Additionally we obtain a representation for the Bergman projection from $L^{2}(\Omega)$ onto $H^{2}(\Omega)$.

\section{Properties of solutions of the Vekua equation}

Let $K \subset \mathbb{C}$ be a compact set. A function $W: K \rightarrow \mathbb{C}$ is called Hölder continuous on $K$ with exponent $0<\alpha<1$ if there exists $M>0$ such that

$$
\left|W\left(z_{1}\right)-W\left(z_{2}\right)\right| \leq M\left|z_{1}-z_{2}\right|^{\alpha}
$$

for all $z_{1}, z_{2} \in K$. The set of such functions is denoted by $C^{\alpha}(K)$. If $\Omega$ is a domain (that is, open and connected) then we define $C^{\alpha}(\Omega)$ to be the space 
of functions $W: \Omega \rightarrow \mathbb{C}$ such that $W \in C^{\alpha}(K)$ for any $K \subset \Omega$ compact.

Consider the Vekua equation

$$
\partial_{\bar{z}} W=a W+b \bar{W}, \quad \text { in } \Omega,
$$

where $\partial_{\bar{z}}=\frac{1}{2}\left(\partial_{x}+i \partial_{y}\right)$ and the coefficients $a, b \in C^{\alpha}(\Omega)$ are bounded functions in $\Omega$. We shall deal with classical solutions of (1).

Proposition 1 ([5, pag 7]) Let $\varphi$ be a bounded measurable function defined on a bounded domain $\Omega \subset \mathbb{C}$. Consider the integral operator

$$
\left(T_{\Omega} \varphi\right)(z):=\frac{1}{\pi} \int_{\Omega} \frac{\varphi(\zeta)}{z-\zeta} d \Omega_{\zeta}
$$

Then

(i) If $|\varphi(z)| \leq M$ in $\Omega$ then $\left|T_{\Omega} \varphi(z)\right| \leq k_{1} M$ for all $z \in \mathbb{C}, k_{1}$ depending only on the area of $\Omega$.

(ii) $T_{\Omega} \varphi(z) \in C^{\alpha}(K)$ for any compact $K \subset \mathbb{C}$ and any $0<\alpha<1$.

(iii) If $\varphi \in C^{\alpha}(\Omega)$, then the first order partial derivatives of $T_{\Omega} \varphi$ belong to $C^{\alpha}(\Omega)$, and $\partial_{\bar{z}} T_{\Omega} \varphi(z)=\varphi(z)$ in $\Omega$.

Proposition 2 Let $W$ be a bounded measurable function in a domain $\Omega$. Set

$$
\Phi=W-T_{\Omega}(a W+b \bar{W}) .
$$

Then, $W$ is a solution of (1) in $\Omega$ iff $\Phi$ is analytic in $\Omega$.

Proof. First note that if $W \in C^{\alpha}(\Omega)$ then from Proposition 1, part (iii), $T_{\Omega}(a W+b \bar{W})$ is continuously differentiable and

$$
\partial_{\bar{z}} T_{\Omega}(a W+b \bar{W})=a W+b \bar{W} .
$$

Assume that $W \in C^{1}(\Omega)$ (thus, $\left.W \in C^{\alpha}(\Omega)\right)$ is a solution of (11). Then application of $\partial_{\bar{z}}$ to $\Phi$ gives $\partial_{\bar{z}} \Phi=\partial_{\bar{z}} W-(a W+b \bar{W})=0$. Thus $\Phi$ is analytic. On the other hand suppose that $\Phi$ is analytic. Then, as $W$ is bounded by Proposition 1, part $(i), T_{\Omega}(a W+b \bar{W}) \in C^{\alpha}(\Omega)$. Since $\Phi \in$ $C^{\alpha}(\Omega)$ it follows that $W \in C^{\alpha}(\Omega)$. Using $\partial_{\bar{z}} \Phi=0$ we get

$$
\partial_{\bar{z}} W=\partial_{\bar{z}} \Phi+\partial_{\bar{z}} T_{\Omega}(a W+b \bar{W})=a W+b \bar{W} .
$$


Proposition 3 Any solution of (1) in $\Omega$ has Hölder continuous partial derivatives in every compact subdomain of $\Omega$.

Proof. This follows from the above proposition and from the properties of the operator $T_{\Omega}$ established in Proposition 1 ,

Proposition 4 The limit of a uniformly convergent sequence of solutions of (1) is a solution of (1) as well.

Proof. Let $W_{n}(z)$ be a sequence of bounded solutions of (11) in $\Omega$ such that $W_{n}(z) \rightarrow W(z)$ uniformly in $\Omega$. It follows from Proposition 2 that the functions $\Phi_{n}$ defined by

$$
\Phi_{n}=W_{n}-T_{\Omega}\left(a W_{n}+b \overline{W_{n}}\right)
$$

are analytic in $\Omega$. Then the uniform limit $\Phi=W-T_{\Omega}(a W+b \bar{W})$ is also an analytic function in $\Omega$. The last equality together with Proposition 2 allows one to conclude that $W$ is a solution of (1) in $\Omega$.

Theorem 5 (Similarity Principle [5], [16]) Let $W$ be a solution of (1) in $\Omega$ (allowed to be unbounded) and set $S:=T_{\Omega}\left(a+\frac{\bar{W}}{W} b\right)$. Then there exists an analytic function $\Psi$ in $\Omega$ such that

$$
W=\Psi e^{S}, \quad \text { in } \Omega .
$$

Remark 6 We stress that $a+\frac{\bar{W}}{W} b$ is a bounded function in $\Omega$ (even if $W$ is unbounded) and thus from part (ii) of Proposition 1 it follows that the function $S$ of the above theorem is Hölder continuous in every bounded domain. Moreover, from part ( $i)$ of the same proposition it is easy to see that $|S(z)|=k_{1} M$ for all $z \in \mathbb{C}$, where $M=\sup _{\Omega}(|a(z)|+|b(z)|)$. This proves that $S$ is bounded by a constant that does not depend on $W$.

\section{The Bergman space for the Vekua equation}

Let $\Omega$ be a bounded domain and $L^{2}(\Omega)$ the classical Lebesgue space of complex valued measurable functions $\varphi$ enjoying the property $\int_{\Omega}|\varphi(z)|^{2} d x d y<$ $\infty$. We define the Bergman space of the Vekua equation (1) as

$$
H^{2}(\Omega)=\left\{W \in C^{1}(\Omega) \cap L^{2}(\Omega): \partial_{\bar{z}} W=a W+b \bar{W} \text { in } \Omega\right\},
$$

where $a, b \in C^{\alpha}(\Omega)$ are bounded functions in $\Omega$. 
It would be convenient to introduce an inner product structure on $H^{2}(\Omega)$, regarding it as a subspace of $L^{2}(\Omega)$. However, since $H^{2}(\Omega)$ is a real linear space it is necessary to consider $L^{2}(\Omega)$ as a real linear space as well. The following remark is useful for what follows.

Remark 7 Let $\mathcal{H}_{\mathbb{C}}$ be a complex linear space and $\langle\cdot, \cdot\rangle_{\mathbb{C}}$ a (complex) inner product defined there. Denote by $\mathcal{H}_{\mathbb{R}}$ the same set (as $\mathcal{H}_{\mathbb{C}}$ ) but understood as a real linear space. Then, it is straightforward to check that $\langle u, v\rangle_{\mathbb{R}}:=$ $\operatorname{Re}\langle u, v\rangle_{\mathbb{C}}$ is a (real) inner product in $\mathcal{H}_{\mathbb{R}}$. Moreover, both scalar products induce the same norm, that is,

$$
\|u\|_{\mathbb{C}}=\sqrt{\langle u, u\rangle_{\mathbb{C}}}=\sqrt{\langle u, u\rangle_{\mathbb{R}}}=\|u\|_{\mathbb{R}} \text { for all } u \in \mathcal{H}_{\mathbb{C}} \equiv \mathcal{H}_{\mathbb{R}}
$$

Also, if $\mathcal{H}_{\mathbb{C}}$ is a complex Hilbert space then $\mathcal{H}_{\mathbb{R}}$ is a real Hilbert space.

From now on, and according to the previous remark, we consider the real Hilbert space $L^{2}(\Omega)$ with the inner product given by

$$
\begin{aligned}
\langle W, V\rangle & =\operatorname{Re} \int_{\Omega} W \bar{V} d x d y \\
& =\int_{\Omega} \operatorname{Re} W \operatorname{Re} V d x d y+\int_{\Omega} \operatorname{Im} W \operatorname{Im} V d x d y
\end{aligned}
$$

The norm and the inner product in $H^{2}(\Omega)$ are those induced by $L^{2}(\Omega)$,

$$
\langle W, V\rangle_{H^{2}(\Omega)}=\operatorname{Re} \int_{\Omega} W \bar{V} d x d y, \quad\|W\|_{H^{2}(\Omega)}:=\|W\|_{L^{2}(\Omega)} .
$$

If $a=b \equiv 0$ then $H^{2}(\Omega)$ coincides with the classical Bergman space of analytic functions which we shall denote by $A^{2}(\Omega)$ (however, and contrary to the classical case, in the present paper $A^{2}(\Omega)$ is understood as a real space).

The next two propositions generalize corresponding well known results for $A^{2}(\Omega)[2,[9]$.

Proposition 8 Let $K \subset \Omega$ be a compact. There is a constant $C_{K}>0$ such that for all $W \in H^{2}(\Omega)$ the following inequality is valid

$$
\sup _{z \in K}|W(z)| \leq C_{K}\|W\|_{H^{2}(\Omega)} .
$$


Proof. Let $K \subset \Omega$ be a compact and let $W \in H^{2}(\Omega)$. From Proposition 5 there exist $\Psi$ an analytic function in $\Omega$ and $S$ a bounded function in $\mathbb{C}$ such that $W=\Psi e^{S}$. It is clear that $\Psi \in A^{2}(\Omega)$. According to Remark 6 there exists a constant $M>0$ independent of $W$ such that $\left|e^{S(z)}\right|,\left|e^{-S(z)}\right| \leq M$, for all $z \in \mathbb{C}$. Hence

$$
\sup _{z \in K}|W(z)| \leq M \sup _{z \in K}|\Psi(z)| .
$$

Moreover, according to [9, Lemma 1.2.1], [16] there exists a constant $D_{K}>$ 0 , depending on $K$, such that

$$
\sup _{z \in K}|\Psi(z)| \leq D_{K}\|\Psi\|_{L^{2}(\Omega)}, \forall \Psi \in A^{2}(\Omega) .
$$

Combining the last inequalities we obtain the desired result

$$
\sup _{z \in K}|W(z)| \leq M D_{K}\|\Psi\|_{L^{2}(\Omega)}=M D_{K}\left\|W e^{-S}\right\|_{L^{2}(\Omega)} \leq M^{2} D_{K}\|W\|_{L^{2}(\Omega)} .
$$

Proposition $9 \mathrm{H}^{2}(\Omega)$ is a Hilbert space. Moreover, the convergence in $H^{2}(\Omega)$ implies the uniform convergence on all compact subsets of $\Omega$.

Proof. Let $W_{n}$ be a Cauchy sequence in $H^{2}(\Omega)$ and let $K$ be a compact subset of $\Omega$. As $W_{n}$ is a Cauchy sequence in the Hilbert space $L^{2}(\Omega)$ there exists $W \in L^{2}(\Omega)$ such that $W_{n} \rightarrow W$ with respect to the $L^{2}$ norm. Using this together with Proposition 8 we see that $W_{n}$ is a Cauchy sequence in the Banach space $C(K)$. Thus, $W_{n}$ converges uniformly on $K$ to some function $\widetilde{W} \in C(K)$. It follows from Proposition 4 that $\widetilde{W}$ is a solution of the Vekua equation. In order to finish the proof let us show that $W=\widetilde{W}$ almost everywhere in $K$. It is easy to see from the above reasoning that $W_{n} \rightarrow W$ in $L^{2}(K)$ as well as $W_{n} \rightarrow \widetilde{W}$ in $L^{2}(K)$. Thus, $W=\widetilde{W}$ as elements of $L^{2}(K)$ and the proposition is proved.

\section{The Bergman kernel for the Vekua equation}

Let $\zeta \in \Omega$. From Proposition 8 it follows that both functionals $\mathcal{R}_{\zeta}, \mathcal{I}_{\zeta}$ acting from $H^{2}(\Omega)$ to $\mathbb{R}$ as $\mathcal{R}_{\zeta} W:=\operatorname{Re} W(\zeta), \mathcal{I}_{\zeta} W:=\operatorname{Im} W(\zeta)$ are continuous. Thus, by Riesz' representation theorem there are functions $K(\zeta, z) \equiv$ $k_{\zeta}(z) \in H^{2}(\Omega)$ and $L(\zeta, z) \equiv l_{\zeta}(z) \in H^{2}(\Omega)$ such that

$$
\operatorname{Re} W(\zeta)=\langle W(z), K(\zeta, z)\rangle_{H^{2}(\Omega)}
$$


and

$$
\operatorname{Im} W(\zeta)=\langle W(z), L(\zeta, z)\rangle_{H^{2}(\Omega)}
$$

for all $W \in H^{2}(\Omega)$. The kernels $K(\zeta, z)$ and $L(\zeta, z)$ enjoy the following interesting relations.

Proposition 10 For any $\zeta, z \in \Omega$ the equalities hold

$$
\operatorname{Re} K(\zeta, z)=\operatorname{Re} K(z, \zeta), \quad \operatorname{Im} L(\zeta, z)=\operatorname{Im} L(z, \zeta)
$$

and

$$
\operatorname{Re} L(\zeta, z)=\operatorname{Im} K(z, \zeta) .
$$

Proof. Let $\zeta_{1} \in \Omega$. Taking $W(z)=K\left(\zeta_{1}, z\right)$ in (2) we obtain $\operatorname{Re} K\left(\zeta_{1}, \zeta\right)=$ $\left\langle K\left(\zeta_{1}, z\right), K(\zeta, z)\right\rangle_{H^{2}(\Omega)}$. Moreover, since $H^{2}(\Omega)$ is a real space

$\operatorname{Re} K\left(\zeta_{1}, \zeta\right)=\left\langle K\left(\zeta_{1}, z\right), K(\zeta, z)\right\rangle_{H^{2}(\Omega)}=\left\langle K(\zeta, z), K\left(\zeta_{1}, z\right)\right\rangle_{H^{2}(\Omega)}=\operatorname{Re} K\left(\zeta, \zeta_{1}\right)$

The other equalities are proved similarly.

Gathering equalities (2) and (3) we obtain

$$
\begin{gathered}
W(\zeta)=\langle W(z), K(\zeta, z)\rangle_{H^{2}(\Omega)}+i\langle W(z), L(\zeta, z)\rangle_{H^{2}(\Omega)} \\
=\int_{\Omega}\{(\operatorname{Re} K(\zeta, z)+i \operatorname{Re} L(\zeta, z)) \operatorname{Re} W(z)+(\operatorname{Im} K(\zeta, z)+i \operatorname{Im} L(\zeta, z)) \operatorname{Im} W(z)\} d x d y .
\end{gathered}
$$

With the aid of Proposition 10 this equality can be written in the form

$$
W(\zeta)=\int_{\Omega}\{K(z, \zeta) \operatorname{Re} W(z)+L(z, \zeta) \operatorname{Im} W(z)\} d x d y .
$$

Definition 11 We define the Bergman kernel of the Vekua equation with coefficient $\alpha \in \mathbb{C}$ and center $\zeta \in \Omega$ as

$$
B(\alpha, \zeta, z):=(\operatorname{Re} \alpha) K(\zeta, z)+(\operatorname{Im} \alpha) L(\zeta, z) .
$$

The previous construction leads to the following statement.

Proposition 12 The Bergman kernel $B(\alpha, \zeta, z)$ belongs to $H^{2}(\Omega)$ in the variable $z$ and enjoys the reproducing property

$$
W(\zeta)=\int_{\Omega} B(W(z), z, \zeta) d x d y, \quad \zeta \in \Omega
$$

for all $W \in H^{2}(\Omega)$. 
Remark 13 Consider the case $a=b=0$, meaning that the Vekua equation defines analytic functions. Hence if $W \in A^{2}(\Omega)$ then $i W \in A^{2}(\Omega)$ and $<W, i V>_{A^{2}(\Omega)}=-\left\langle i W, V>_{A^{2}(\Omega)}\right.$. Using this together with (2) and (3) it is easy to see that

$$
\operatorname{Re} L(\zeta, z)=-\operatorname{Re} L(z, \zeta), \quad \operatorname{Im} L(\zeta, z)=\operatorname{Re} K(z, \zeta) .
$$

On the other hand, from the last equalities and Proposition 10 it follows that $K(z, \zeta)=-i L(z, \zeta)$. Substituting this into (4) gives us the classical Bergman reproducing formula [3]

$$
W(\zeta)=\int_{\Omega} K(z, \zeta) W(z) d x d y
$$

\section{Construction of the Bergman kernel by means of a countable orthonormal system. The Bergman projection}

Since $H^{2}(\Omega)$ is a closed subspace of $L^{2}(\Omega)$ then

$$
L^{2}(\Omega)=H^{2}(\Omega) \oplus\left(H^{2}(\Omega)\right)^{\perp}
$$

and there exists an orthogonal projection $P_{\Omega}$ from $L^{2}(\Omega)$ onto $H^{2}(\Omega)$. $P_{\Omega}$ is named the Bergman projection.

Proposition 14 Let $\varphi \in L^{2}(\Omega)$. Then

$$
\left(P_{\Omega} \varphi\right)(\zeta)=\int_{\Omega} B(\varphi(z), z, \zeta) d x d y, \quad \zeta \in \Omega
$$

Proof. From (22), (3) and by Proposition 10 it is straightforward to see that

$$
\int_{\Omega} B(\varphi(z), z, \zeta) d x d y=\langle\varphi(z), K(\zeta, z)\rangle_{H^{2}(\Omega)}+i\langle\varphi(z), L(\zeta, z)\rangle_{H^{2}(\Omega)} .
$$

Let $Q_{\Omega}$ be the operator defined on $L^{2}(\Omega)$ by the right-hand side of this equality. According to (6), if $\varphi \in L^{2}(\Omega)$ there exist $W \in H^{2}(\Omega)$ and $\psi \in$ $\left(H^{2}(\Omega)\right)^{\perp}$ such that $\varphi=W+\psi$. Since $\psi$ is orthogonal to both $K(\zeta, z)$ and $L(\zeta, z)$ (both kernels belong to $H^{2}(\Omega)$ ) then $Q_{\Omega} \psi=0$ and hence

$$
Q_{\Omega} \varphi=Q_{\Omega}(W+\psi)=Q_{\Omega} W+Q_{\Omega} \psi=Q_{\Omega} W
$$


This shows that $Q_{\Omega}^{2}=Q_{\Omega}$ and that the range of $Q_{\Omega}$ is $H^{2}(\Omega)$, or equivalently, $Q_{\Omega}$ is the orthogonal projection from $L^{2}(\Omega)$ onto $H^{2}(\Omega)$. Hence $Q_{\Omega}=P_{\Omega}$ and the proof is finished.

A subset $M$ of a normed linear space $X$ is called complete if $\operatorname{span} M$ is dense in $X$. Obviously, if $\mathcal{H}$ is a Hilbert space then from a countable and complete subset of $\mathcal{H}$ it is possible to construct a countable orthonormal basis of $\mathcal{H}$, first removing the linearly dependent elements and then applying the Gram-Schmidt orthonormalization process. Moreover, under the notation of Remark 7 it is clear that if $\left\{\varphi_{n}\right\}_{n \in \mathbb{N}}$ is a complete subset of $\mathcal{H}_{\mathbb{C}}$ then $\left\{\varphi_{n}, i \varphi_{n}\right\}_{n \in \mathbb{N}}$ is a complete subset of $\mathcal{H}_{\mathbb{R}}$.

Remark 15 If $\Omega$ is a bounded simply connected domain whose boundary is also the boundary of an infinite region then the system of the usual nonnegative powers $\left\{z^{n}, i z^{n}\right\}_{n \in \mathbb{N}_{0}}$ is complete in $A^{2}(\Omega)$ [8], [12].

Proposition $16 H^{2}(\Omega)$ has a countable complete subset.

Proof. It is well know that $L^{2}(\Omega)$ has a countable orthonormal basis $\left\{\varphi_{n}\right\}_{n \in \mathbb{N}}$ (see [13]). Thus, given $W \in H^{2}(\Omega) \subset L^{2}(\Omega)$ we can write the corresponding Fourier series $W=\sum_{n=1}^{\infty} c_{n} \varphi_{n}$ with $c_{n}=\left\langle W, \varphi_{n}\right\rangle_{L^{2}(\Omega)}$. Since $P_{\Omega}$ is a bounded operator and a projection onto $H^{2}(\Omega)$, after applying $P_{\Omega}$ to both sides of the above series we get $W=P_{\Omega} W=\sum_{n=1}^{\infty} c_{n} P_{\Omega} \varphi_{n}$. This means that $\operatorname{span}\left\{P_{\Omega} \varphi_{n}\right\}_{n \in \mathbb{N}}$ is dense in $H^{2}(\Omega)$. The statement is proved.

Proposition 17 Let $\left\{\varphi_{n}\right\}_{n \in \mathbb{N}}$ be an orthonormal basis of $H^{2}(\Omega)$ and $W \in$ $H^{2}(\Omega)$. Then,

$$
W(z)=\sum_{n=1}^{\infty}\left\langle W, \varphi_{n}\right\rangle_{H^{2}(\Omega)} \varphi_{n}(z) .
$$

The series converges in $H^{2}(\Omega)$ with respect to the variable $z$ and uniformly on compact subsets of $\Omega$. In particular,

$$
B(\alpha, \zeta, z)=\sum_{n=1}^{\infty}\left[\operatorname{Re}\left(\bar{\alpha} \varphi_{n}(\zeta)\right)\right] \varphi_{n}(z) .
$$

Proof. Formula (7) is the representation of $W$ as a Fourier series corresponding to the orthonormal basis $\left\{\varphi_{n}\right\}$, thus it is convergent in $H^{2}(\Omega)$. The uniform convergence then follows due to Proposition 9, The last part of the statement follows from (7) taking $W(z)=B(\alpha, \zeta, z)$. 
Remark 18 An analogue of the Runge theorem from complex analysis is available for the Vekua equation, replacing the usual powers of complex analysis by a special countable system of solutions of the Vekua equation called formal powers [5]. There are general conditions under which the system of formal powers can be constructed by a simple algorithm [5], [11], [7], [6]. The previous proposition is obviously related to the Runge theorem due to uniform convergence on compact sets. We conjecture that if $\Omega$ is a Jordan domain then the system of nonnegative formal powers is complete in $H^{2}(\Omega)$.

Remark 19 The requirement about the smoothness of the coefficients a and $b$ of equation (1) was imposed in order to simplify the exposition. If $a, b \in$ $L^{p}(\Omega), p>2$, and dealing with weak solutions of (1), Proposition 4 and Theorem 5 (the ones used in Section [3) keep to be valid [16]. Moreover, weak solutions of (1) are Hölder continuous and thus the study of the functionals $\mathcal{R}_{\zeta} W:=\operatorname{Re} W(\zeta), \mathcal{I}_{\zeta} W:=\operatorname{Im} W(\zeta)$ makes sense (note that this was the starting point in Section 4 to establish the existence of the Bergman kernel).

\section{References}

[1] Aronszajn N 1950 Theory of reproducing kernels. Trans. Am. Math. Soc. 68, 337-404.

[2] Bell S 1992 The Cauchy transform, potential theory, and conformal mapping, CRC Press, Boca Raton.

[3] Bergman S 1970 The Kernel Function and Conformal Mapping, Am. Math.Soc., Providence, RI.

[4] Bergman S and Schiffer M 1953 Kernel Functions and Elliptic Differential Equations in Mathematical Physics. Academic Press, New York.

[5] Bers L 1952 Theory of pseudo-analytic functions. New York University.

[6] Campos H M and Kravchenko V V 2013 Fundamentals of bicomplex pseudoanalytic function theory: Cauchy integral formulas, negative formal powers and Schrödinger equations with complex coefficients. Complex Anal. Oper. Theory, v. 7, issue 2, 485-518.

[7] Campos H M., Castillo, R and Kravchenko, V.V 2012 Construction and application of Bergman-type reproducing kernels for boundary and eigenvalue problems in the plane. Complex Var. Elliptic Equ. 57(7-8),787-824. 
[8] Farrell O 1934 On approximation to analytic functions by polynomials, Bulletin of the American Mathematical Society, vol. 40 , pp. 908-914.

[9] Krantz S 2007 Geometric Function Theory: Explorations in Complex Analysis, Cornerstones, Birkhäuser Boston.

[10] Krantz S 2013 Geometric Analysis of the Bergman Kernel and Metric. Graduate Texts in Mathematics, 268. New York Springer.

[11] Kravchenko V V 2009 Applied Pseudoanalytic Function Theory, Birkhäuser, Basel.

[12] Markushevich A L 1965 Theory of functions of a complex variable, vol. 1. Prentice-Hall, Englewood Cliffs, New Jersey.

[13] Reed M and Simon B 1980 Methods of Modern Mathematical Physics I. Functional Analysis, rev. and enl. edition, Academic Press, San Diego.

[14] Saitoh S 1988 Theory of Reproducing Kernels and Its Applications. Pitman Research Notes in Mathematics Series, vol. 189.

[15] Saitoh S and Sawano Y 2016 Theory of Reproducing Kernels and Applications. Developments in Mathematics, vol. 44. Springer, Berlin.

[16] Vekua I N 1959 Generalized analytic functions. Moscow: Nauka (in Russian); English translation Oxford: Pergamon Press 1962. 